\section{Denn sie wissen nicht, was sie tun?}

\author{
International wird die Forderung nach einer Verbesserung der Verbraucher-infor- \\ mation stärker, vor allem innerhalb des "Consumer Policy Service" der Europäi- \\ schen Kommission oder bei "Consumers International", dem weltweiten Dachver- \\ band aller Verbraucherorganisationen. Werden sich Konsumenten aufgrund von \\ mehr Information nachhaltiger, also sozial- und umweltverträglicher verhalten?
}

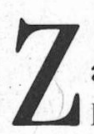
Von Cornelia Weskamp ahlreiche Untersuchungen belegen, daß Konsumenten sich mehr Informationen über Produkte und ihre Eigenschaften wünschen (1). Dabei wird davon ausgegangen, daß zwischen Produzenten und Konsumenten ein immenses Informationsgefälle besteht, man spricht von asymmetrischer Informationsverteilung (2). Da produktspezifische Informationen gerade im Zuge des Herstellungsprozesses gewonnen werden, könnten sie leicht von den Produzenten zur Verfügung gestellt werden. Bei komplexen Stoff- und Akteursketten gehen diese Informationen jedoch oftmals ganz oder teilweise verloren. Darüber hinaus ist der Produzent aus drei Gründen als Informationslieferant für die Konsumenten untauglich:

1. Produzenten haben keinen Anreiz, auch negative Eigenschaften ihrer Produkte offenzulegen.

2. Die Informationen über Produkteigenschaften sind für Konsumenten nicht ohne externe Verifikation glaubwürdig (3).

3. Eine Offenlegung aller Produktinformationen führt zu einer Informationsüberlastung der Konsumenten.
Ohne hinreichende Information dagegen entspricht die Nachfrage nach einem Produkt nur zufällig den Präferenzen des Konsumenten. Es kommt zu Marktversagen durch Informationsasymmetrie. Damit wird Phänomenen wie der sogenannten adversen Selektion der Boden bereitet (4). Adverse Selektion bedeutet, daß Produzenten von Produkten mit höherer Qualität keinen Vorteil gegenuiber Wettbewerbern haben, deren Produkte vergleichsweise schlechtere Eigenschaften aufweisen, da die Nachfrager die angebotenen Qualitäten nicht unterscheiden können. Sie zeigen daher auch keine höhere Zahlungsbereitschaft für mehr Qualität. Adverse Selektion tritt also ein, wenn Konsumenten Produkteigenschaften selbst nicht aufdecken oder wenn Produzenten Qualitätsvorteile nicht glaubhaft signalisieren können: Als Folge bieten die Produzenten keine überdurchschnittliche Qualität mehr an, da sie gegenüber Billiganbietern keine Absatzvorteile sondern nur Kostennachteile haben (5). Dies kann auch den Effekt haben, daß Anbieter vorrangig in beobachtbare Produktmerkmale investieren, die Qualitätseigenschaften suggerieren sollen.

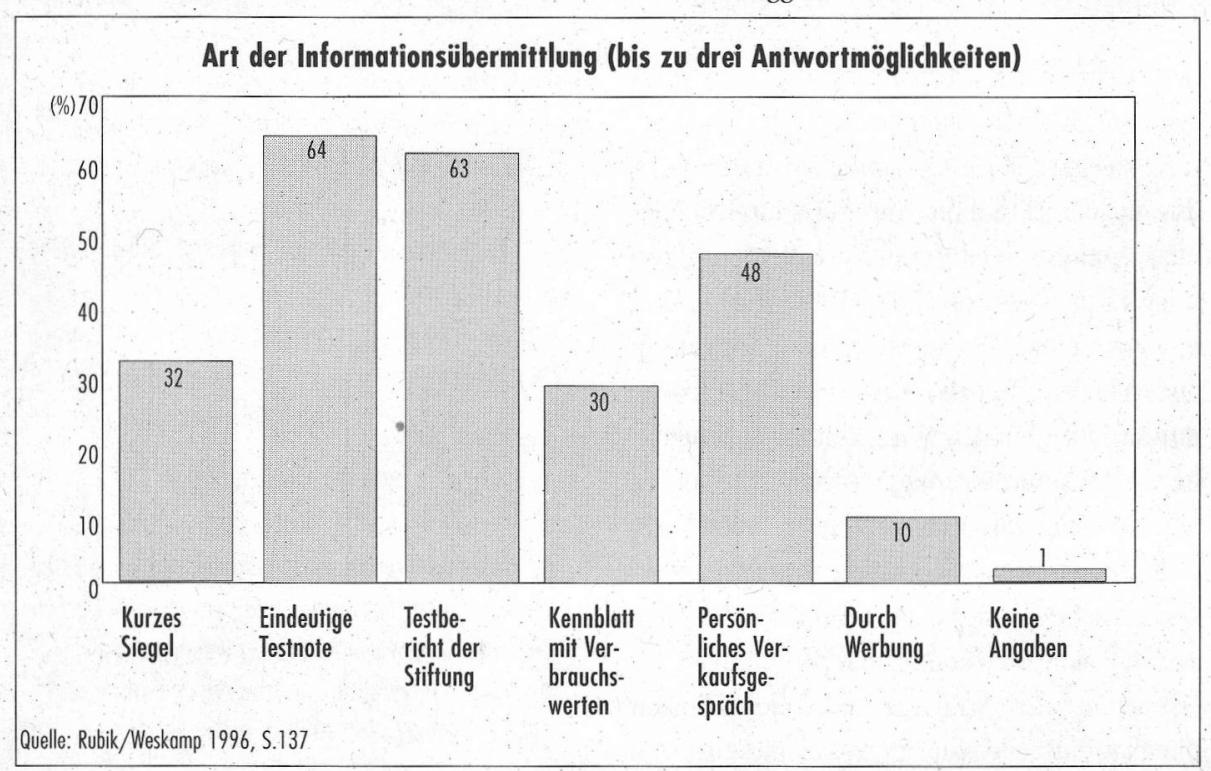

In bezug auf die Qualitäts-, Umwelt- oder Gesundheitseigenschaften von Produkten macht das eine glaubwürdige Kommunikation der Vertrauenseigenschaften notwendig. Diese kann negativen Auswirkungen wie adverser Selektion begegnen und spielt eine bedeutende Rolle für die Funktionsfähigkeit der Märkte.

\section{Informationskosten}

Bei der Beschaffung und Verarbeitung der Information entstehen dem Konsumenten allerdings verschiedene Arten von Kosten:

- finanzielle (für Zeitschriften, Kopien, Telefonate, Beratungsdienste usw.),

- zeitliche (Opportunitätskosten in Form von Freizeitverlust),

- kognitive (Auswahl der relevanten Informationsquellen, Aufnahme und Verarbeitung der Information) und

- physische (körperliche Anstrengung bei Wegen).

Konsumenten kaufen oft nur relativ kleine Mengen einer Vielzahl von Produkten, manche Kaufentscheidungen stehen nur ein bis zweimal im Leben an, manche jeden Tag. Bei jeder Kaufentscheidung alle notwendigen Informationen zu beschaffen und eine gründliche Prüfung der Produkteigenschaften vorzunehmen, wäre $\mathrm{zu}$ aufwendig. Darüber hinaus steht bei Nutzung, Ge- und Verbrauch bzw. Entsorgung der Produkte wiederum eine Vielzahl von Entscheidungen an, die eine Informationssuche notwendig machen. Informationen sind somit im Sinne der Informationsökonomik als eigenständiges Gut zu betrachten, dessen Gewinnung mit Informationskosten verbunden ist (6).

\section{Informationsselektion}

Die Intensität der Informationssuche hängt von dem Risiko ab, das der Konsument subjektiv mit dem Kauf verbindet. Je höher er dieses Risiko einschätzt, desto mehr wird er bereit sein, die verborgenen Eigenschaften offenzulegen und Transaktionskosten für zusätzliche Informationen in Kauf zu nehmen (7). Auf der anderen Seite muß ihm die soziale und ökologische Relevanz einer Kauf- oder Nutzungsentscheidung deutlich sein, um auf Informationssuche zu gehen. Mit anderen Worten: Es ist eine gewisse Vorinformation notwendig, um die nötige Motivation aufzubringen, in speziellen Situationen auf zusätzliche Informationen zurïckgreifen zu wollen. Erschwerend kommt bei der Ausbildung eines solchen Umweltbewußtseins 
hinzu, daß Umweltschäden von den Konsumenten oft nicht unmittelbar wahrgenommen werden können, sondern nur über Medien wie Bilder, Texte, Meßgeräte vermittelt werden. Sie sind damit in Alltagssituationen nicht präsent und können leicht verdrängt werden.

Für Konsumenten umweltverträglicher Produkte sind darüber hinaus die Transaktionskosten vergleichsweise hoch, weil die Informationsasymmetrie und die Unsicherheit im Vergleich zu herkömmlichen Produkten besonders hoch sind.

Ein Konsument wird aber nur solange Informationen beschaffen, d.h. Informationskosten auf sich nehmen, wie er sich davon einen zusätzlichen Nutzen verspricht. Daher liegt der Nutzen einer Produktinformation für den Konsumenten in der Senkung informatorischer Kosten. Dabei muß berücksichtigt werden, daß der Erfolg der Informationssuche aus Sicht des Konsumenten ungewiß ist.

In einer repräsentativen Umfrage votierten die Konsumenten eindeutig fïr Produktinformationen, denen eine Bewertung von Experten vorausgegangen išt, und die gleichzeitig einen Vergleich verschiedener Produkte ermöglichen. So wurden mit Abstand am häufigsten die Testnote und der Testbericht der Stiftung Warentest gewählt, wenn es um Informationsübermittlung beim Waschmaschinenkauf ging (vgl. Abb)

\section{- Weniger ist mehr !}

Die Grenzen für die Ausbildung nachhaltiger Konsummuster durch verbesserte Verbraucherinformation werden durch folgende Faktoren bestimmt: Möchte ein Konsument überhaupt auf Informationssuche gehen und in welcher Höhe ist er bereit, Transaktionskosten in Kauf zu nehmen? Wieviel zusätzliche Verbraucherinformationen könnenr Konsumenten berücksichtigen? Eine Verbesserung des Informationsstandes der Verbraucher anzustreben, kann nicht bedeuten, nur zusätzliche Informationen zu produzieren. Kauf- und Nutzungsentscheidungen sind für die Konsumenten oft mit einer Flut von widersprüchlichen Informationen verbunden. Alle relevanten Informationen zur Beurteilung von Produkten und ihren Eigenschaften zusammenzutragen, ist nahezu aussichtslos (8) und würde zu einer Überforderung des Konsumenten führen (information overload). Empirische Untersuchungen, wie von Jacoby et al., kamen zu dem Ergebnis, daß ein Zusammenhang zwischen Informationsmenge und Entscheidungseffizienz besteht. Die Entscheidungspersonen mit mehr Informationen fuihlten sich zwar subjektiv besser, trafen allerdings die schlechteren Kaufentscheidungen. Im Durchschnitt kann eine Person etwa sieben Produkteigenschaften, wie Preis, Gewicht, Material etc., bei einer Kaufentscheidung verarbeiten. Bei deutlich mehr Eigenschaften wird diese Informationen nur noch selektiv wahrnehmen.

Zum Schutz vor einer Reizüberflutung der Konsumenten sind daher Instrumente wie Produktkennzeichen entwickelt worden, die Konsumenten mit vereinfachter Information über eine Fülle von Produktmerkmalen und die Auswirkungen, die von Produkten ausgehen, in Kenntnis setzen. Allerdings ist die Reduktion auf solche Schlüsselinformationen immer eine Gratwanderung zwischen der gewünschten Reduktion von komplexen Informationen und der unerwünschten Abhängigkeit von Expertenwissen.

\section{Anforderungen an eine verbes- serte Verbraucherinformation}

Folgende Anforderungen an eine verbesserte Verbraucherinformation sind (9) formulierbar:

- Angebot von vorbewerteter, leicht verständlicher Information,

- Beschränkung auf entscheidungsrelevante Informationen,

- Differenzierung nach einzelnen Qualitätsfaktoren oder Teilqualitäten,

- Unterscheidung nach informativer Produktkennzeichnung und anderen Informationen,

- Verläßlichkeit und Glaubwürdigkeit der Verbraucherinformation,

- hoher Bekanntheitsgrad,

- Transparenz und Vergleichbarkeit der Produktalternativen,

- vollständige Markterfassung,

- Praktikabilität für Produzenten, Handel und Kontrollinstanz.

Konsumenten sind eine heterogene Gruppe mit unterschiedlichen Interessen und Eigenarten. Eine Verbraucherinformation muß diese Heterogenität berücksichtigen und die verschiedenen Informationsbedürfnisse auf unterschiedliche Art und Weise befriedigen. Dazu ist möglicherweise ein duales System geeignet: das heißt eine Kombination von vorbewerteter komparativer Produktinformation und der Möglichkeit, ausfiuhrliche Produktinformationen anzufordern (10). Inwieweit hier mögliche Qualitätsstandards von Verbraucherinformationen sinnvoll sind, die eine Vereinheitlichung in bezug auf Glaubwürdigkeit, Verständlichkeit, Bekanntheitsgrad etc. gewährlei-

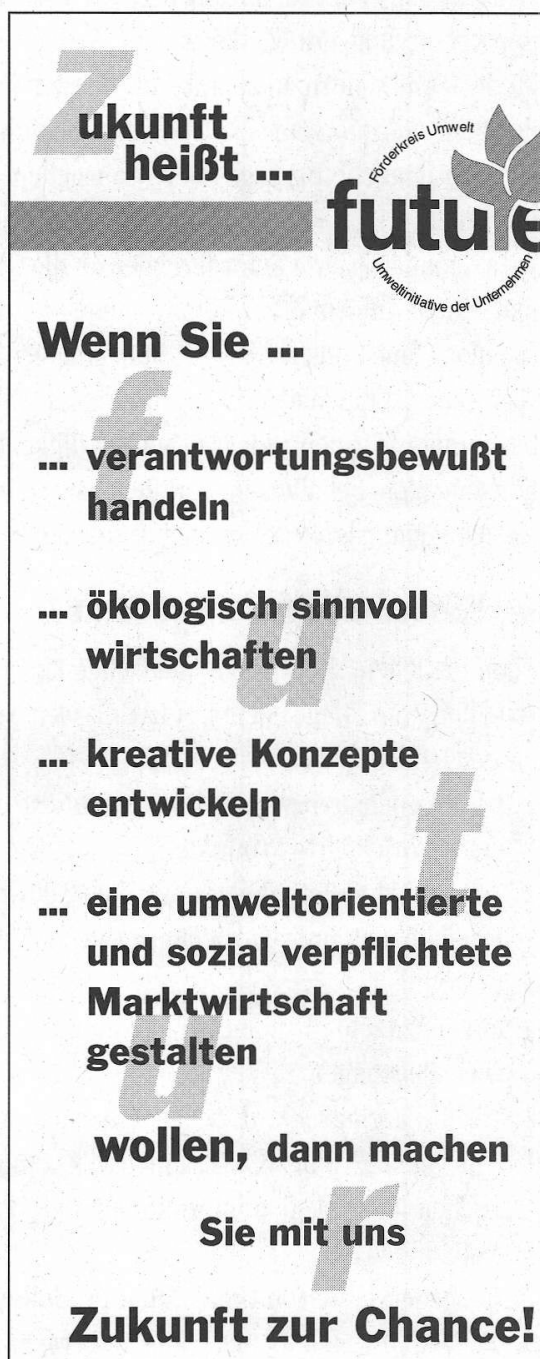

Denn der Förderkreis Umwelt future e.V. - eine Umweltinitiave engagierter Unternehmer steht für einen effektiven betrieblichen Umweltschutz und entwickelt die dazu notwendigen Instrumente.

Ja, senden Sie uns

O ausführliche Informationen

O ein Probeexemplar der futureZeitschrift "Unternehmen und Umwelt"

Firma

PLZ/Ort

Straße/Nr.

Tel./Ansprechpartner

Förderkreis Umwelt future e.V.

Kollegienwall 22 a

49074 Osnabrück

Tel.: 0541/2 8784

Fax:: 0541/2 8304 
sten würden, bleibt zu untersuchen. Die Informationsversorgung erfolgt durch eine Vielfalt von Medien und Informationsträgern. Bislang werden die Konsumenten jedoch durch oftmals widersprüchliche Beiträge eher verunsichert als informiert (11). Koordination und möglicherweise standardisierte Anforderungen an die Qualität einer Information könnten hier Abhilfe schaffen. Eine weiteres Mittel, Konsumenten zu verwirren, ist die Aufdeckung von Bio-Skandalen bestimmter Firmen oder Label-Streitigkeiten. Konsumenten behalten in der Regel nur eines zurück: Echtheitszweifel an allen Bio-Produkten.

\section{- Wissen allein genügt nicht !}

Neun Ursachen, die einer ökologischen Kaufentscheidung im Wege stehen (12), lassen sich unterscheiden:

1. Preisvorbehalt bzw. die höheren Preise für umweltorientierte Produkte,

2. Zugänglichkeitsmühe bzw. die schwere Erhältlichkeit umweltorientierter Produkte,

3. Gewohnheitsbarriere,

4. Effizienznachteil mancher Produkte oder Dienstleistungen,

5. Irrelevanzeindruck, d. h. der Eindruck, daß dem eigenen Konsum gesamtgesellschaftlich gesehen keine Bedeutung zukommt,

6. Ästhetikdefizit,

7. Imagebelastung von Handel und Produkten,

8. Echtheitszweifel, Befürchtung ungerechtfertigt hoher Preise,

9. Minderschätzung künftiger Ereignisse und Bedürfnisse.

Nur wenige Barrieren ließen sich durch Informationsverbesserung ausräumen. Einen geringen Beitrag kann die Verbraucherinformation auch der Zugänglichkeitsmühe (durch z. B. Einkaufsfiuhrer, Adressenlisten) oder der Imagebelastung entgegensetzen. Die anderen Hemmnisse sind durch eine alleinige Verbesserung der Verbraucherinformation nicht zu beseitigen. Es sind vielmehr die Anreize für sozial-ökologisches Verhalten, die fehlen (13).

Unabhängig davon, ob man den Konsumenten als homo oeconomicus betrachten möchte oder als beschränkt rational handelndes Wesen: Sich beim Kauf oder der Nutzung eines Produktes nach dem Preis oder Aussehen zu richten, wenn alle anderen Produkteigenschaften nur unter großen Mühen herauszufinden wären, ist in gewissem Sinne rational.

Dabei würden die meisten Konsumenten Mehrkosten und Mühen für eine intakte Umwelt und
Gesellschaft in Kauf nehmen, sie können sie jedoch durch individuelles Markthandeln nicht herbeiführen. Ein direkter umweltbezogener Nutzen von Produkten wie reinere Luft oder sauberes Wasser kann vom Käufer nicht internalisiert werden, weil die Umwelt ein kollektives Gut ist (14). So ist der Grund für den Kauf von Lebensmitteln aus kontrolliert biologischem Anbau denn auch eher die Förderung der eigenen Gesundheit als die Erhaltung der Umwelt. Die Lösung der Umweltproblematik kann jedoch auch nicht erfolgen, indem von den Konsumenten selbstloses, aus der sozialen Vernunft geborenes Handeln und Verzichten gefordert wird, wenn allen anderen Akteuren die Legitimation zu Gewinnstreben und Positionsbehauptung zugestanden wird. Daraus folgt, daß bestimmte umweltpolitische Ziele nur durch ökonomische Anreize und ordnungsrechtliche Maßnahmen erreicht werden können.

Konsumenten sind eine zu große Schar, als daß sich ein gemeinsames Ziel ohne Zwang und selektive Anreize verfolgen ließe. Daher kommt bei Konsumenten auch schnell der Eindruck auf, ihr individuelles Tun sei irrelevant. In einer kleinen Gruppe, z. B. einer Bürgerinitiative oder regionalen Gemeinschaft, kann es durchaus solche kollektiven Handlungen mit großem Erfolg geben (15).

\section{Was ist zu ändern ?}

Die Informationsangebote an Konsumenten müssen qualitativ wachsen, um ökologisches Handeln zu ermöglichen. Eine willkürliche quantitative Ausweitung des Informationsangebotes führt eher zu Verwirrung der Konsumenten und ist kontraproduktiv. Sinnvoll ist daher eher eine komprimierte und komparative Verbraucherinformation, auch wenn eine Verbesserung der Verbraucherinformation vermutlich nur einen geringen Teil zu nachhaltigerem Konsum beitragen kann. Denn auch einem Konsumenten, für den jede Information verfügbar wäre, stehen zahlreiche Verhaltensbarrieren entgegen. Es ist anzunehmen, daß Verhaltensänderungen eher durch positive Anreize und konkrete Vorbilder zu erreichen sind denn durch globale Appelle und detailgetreue Informationen. Hier gibt es noch viel von der psychologischen Disziplin abzuschauen: Kognitiv vermitteltes Wissen führt nur mit geringem Erfolg zu erwünschtem Verhalten. Es sind vielmehr über Vorbilder vermitteltes Beobachtungslernen oder durch Belohnung verstärktes Verhalten, die zu erwünschten nachhaltigen Verhaltensweisen führen.

\section{Anmerkungen:}

1) INRA (International Research Associates): Eurobaro meter 37.1 Consumer Product Labelling. Brüssel 1992 - Fuchslocher, H.: Verbraucher im Spannungsfeld zwischen Modeansprüchen und Umweltinteressen. In cpd Düsseldorf (Hisg): Fashion and ecology symposium. 1995

- Rubik, F./Weskamp, C. : Verbraucherschutz durch Produktkennzeichnung. IÖW-Schriftenreihe Nr. 98/96, Berlin 1996

2) Fritsch, M./Wein, I./Ewers, H.-J.: Marktversagen und Wirtschaftspolitik. Vahlen, München 1993, S. 186

3). Als ein weiterer Grund könnte angeführt werden, daß teilweise die Informationen nicht erschlossen werden können oder sollen; in diesem Fall spricht man jedoch nicht von Informationsasymmetrie.

4) Sinn, H.: Verbraucherschutz als Problem asymmetrischer Informationskosten. Volkswirtschaftliche Fakultät der Ludwig-Maximilian-Universität München - Diskussionspapier 1988, Nr. 88-06, S. 2

5) Akerlof, G.: The Market for 'Lemons': Qualitative Uncertainty and the Market Mechanism. In: Quarterly Journal of economics. 1970, Vol. 84, S. 488-500. Hier wird der Fal adverser Selektion anhand eines Gebrauchtwagenmarktes erläutert.

6) Frey, D./Kumpf, M./Raffée, H./Sauter, B./Silberer, G.: Informationskosten und Reversibilität des Entschlusses als Determinanten der Informationsnachfrage vor Entscheidungen. In: Zeitschrift für experimentelle und angewandte Psychologie. 1976, Heft 4, S. 569-585

7) Nach Thorelli (1975) sind 15 Prozent der Konsumenten Informationssucher (zitiert nach Wendorf, G.: Umweltzeichen im Spannungsfeld zwischen Konsumenten und Unternehmen. Frankfurt a.M. 1994, S.55).

8) vgl. 1) Rubik/Weskamp 1996

9) Gruber, S.: Verbraucherinformation durch Gütezeichen.

Köln U.a.: Heymanns, 1987

- BEUC <Bureau Européen des Unions de Consommateurs> (1994): Camera labelling Voluntary information scheme. Brüssel: Report of the pilot project working group set up by the Consumer Policy Service of the EC Commission.

10) vgl. 8) Kap. 10

11) Kaas, K.P.: Marketing für umwelffreundliche Produkte. In: Die Betriebswirtschaft, JG. 52, Nr. 4, 1992, S. $473 f f$

12) Bänsch, A.: Marketingfolgerungen aus Gründen für den Nichtkauf umwelffreundlicher Konsumgüter. In: Jahrbuch der Absatz- und Verbraucherforschung, 1990, Nr. 4

13 ) Weskamp, C.: Determinanten nachhaltigen Konsums. In: Weskamp, C. (Hrsg): Ökologischer Konsum. Ein Beitrag des Ökoforums, I0̈W, Berlin 1995

14 ) vgl. 11) S. 474

15) vgl. 11) S. 483

Die Autorin

Cornelia Weskamp isi wiss. Mitarbeiterin am lÖW, Regionalbüro NRW, Leilerin des Forschungsfeldes: Ökologischer Konsum

Kontakt: Tel. (0202) 805 30, Fax 83402 
(c) 20I0 Authors; licensee IÖW and oekom verlag. This is an article distributed under the terms of the Creative Commons Attribution Non-Commercial No Derivates License (http://creativecommons.org/licenses/by-nc-nd/3.o/), which permits unrestricted use, distribution, and reproduction in any medium, provided the original work is properly cited. 